\title{
EchoGéo
}

$34 \mid 2015$

Varia

\section{Les périurbains franciliens : vers de nouveaux comportements de mobilité ?}

\section{Martine Berger, Mireille Bouleau et Catherine Mangeney}

\section{OpenEdition}

12 Journals

Édition électronique

URL : https://journals.openedition.org/echogeo/14399

DOI : 10.4000/echogeo.14399

ISSN : 1963-1197

Éditeur

Pôle de recherche pour l'organisation et la diffusion de l'information géographique (CNRS UMR 8586)

Référence électronique

Martine Berger, Mireille Bouleau et Catherine Mangeney, «Les périurbains franciliens : vers de nouveaux comportements de mobilité ? », EchoGéo [En ligne], 34 | 2015, mis en ligne le 15 décembre 2015, consulté le 01 août 2021. URL : http://journals.openedition.org/echogeo/14399 ; DOI : https:// doi.org/10.4000/echogeo.14399

Ce document a été généré automatiquement le 1 août 2021.

EchoGéo est mis à disposition selon les termes de la licence Creative Commons Attribution - Pas d'Utilisation Commerciale - Pas de Modification 4.0 International (CC BY-NC-ND) 


\title{
Les périurbains franciliens : vers de nouveaux comportements de mobilité ?
}

\author{
Martine Berger, Mireille Bouleau et Catherine Mangeney
}

1 De nombreux travaux ont souligné le rôle croissant des territoires de résidence dans les comportements de mobilité des ménages, et la spécificité de l'aire urbaine de Paris, dont les distances et les durées moyennes de déplacements plus élevées s'expliquent par l'étendue, la densité et le niveau de congestion, mais aussi les profils sociodémographiques de ses habitants (Gallez et Orfeuil, 1998 ; Armoogum et al., 2010). Ainsi les habitants des espaces périurbains sont le plus souvent décrits comme parcourant des distances très longues pour se rendre à leur travail, effectuer leurs achats, fréquenter des équipements et services d'usage courant, ou pour leurs loisirs (Baccaïni et al., 2007 ; Hubert et Delisle, 2010 ; Grimal, 2012). À ce titre, les modes de vie périurbains, générateurs de déplacements quotidiens dont l'essentiel est réalisé en automobile et dont la portée tend à augmenter avec la taille des aires urbaines, contribuent fortement à l'augmentation des émissions de $\mathrm{CO}_{2}$ liées à la mobilité (Nicolas et al., 2012).

2 Les périurbains sont souvent cités en exemple de ménages aux territoires quotidiens très distendus, éclatés entre la ville-centre où se concentrent emplois et services, et des résidences situées dans des communes peu denses, possédant au mieux des équipements de base (l'école élémentaire par exemple). Les inflexions récentes des pratiques de mobilité dans l'aire urbaine parisienne conduisent à nuancer ce constat. Dans un premier temps, en nous appuyant principalement sur des sources censitaires, nous analyserons les navettes domicile-travail des habitants des franges de l'agglomération parisienne et des couronnes périurbaines au-delà des limites de la région Île-de-France, jusqu'aux pôles urbains secondaires du Bassin parisien proche, en prenant comme exemple l'ouest francilien au sens large: deux départements de la Grande couronne, Yvelines et Val-d'Oise, et les cantons qui les bordent dans l'Eure, l'Eure-et-Loir et l'Oise, dans un rayon de 20 à $100 \mathrm{~km}$ de Paris (cf. illustration 1) ${ }^{1}$. Dans 
un second temps, à partir des résultats des Enquêtes Globales Transport, nous nous intéresserons aux autres types de déplacements pour interroger la spécificité des pratiques des périurbains en les replaçant dans le cadre de l'ensemble de la région Îlede-France ${ }^{2}$.

3 L'ouest francilien se caractérise à la fois par l'ancienneté de la périurbanisation, qui démarre dès la fin des années 1960, portée par le déplacement du centre de gravité des emplois vers l'ouest et par l'existence d'un semis dense de centres secondaires: les bourgs, les villes petites et moyennes hors agglomération parisienne y sont plus nombreux, mieux répartis et plus rapprochés que dans l'est de l'île-de-France, à la fois du fait d'un héritage de lieux centraux plus nombreux, et de leur renaissance avec la densification de la population (Cavard, 1989; Desplanques, 1990). La spécificité de ce territoire dont la périurbanisation a été l'une des plus précoces de France met en lumière un avenir possible de recomposition des pratiques pour d'autres territoires périurbains plus récents, comme ceux de la Seine-et-Marne ou d'autres régions françaises (Cordobès et al., 2010 ; Vanier, 2011).

Qu'en est-il des mobilités quotidiennes des périurbains de l'ouest de l'île-de France et des cantons riverains, à un moment où on observe une stabilisation du front de périurbanisation, avec une forte décélération de la construction neuve ${ }^{3}$ ? Le solde migratoire des couronnes périurbaines, bien qu'encore positif, y a été divisé par 5 entre 1975-1989 et 1990-2006. On y observe à la fois une densification de la population résidente et des emplois, une réduction sensible des mobilités résidentielles ${ }^{4}$ et un ancrage des populations, contribuant à une meilleure connaissance de l'environnement local et de ses ressources, qui peut conduire à une pratique des territoires voisins plus importante que celle des premiers installés, qui étaient plus souvent, dans les années 1970 ou 1980, originaires de Paris ou de la banlieue proche. Dans le même temps, avec l'accroissement de la population, l'offre de commerces et de services de proximité s'est étoffée, quantitativement et qualitativement.

5 Tant les navettes domicile-travail que les autres types de mobilités quotidiennes conduisent à nuancer l'idée d'une spécificité des mobilités périurbaines, et à souligner les continuités entre les franges de l'agglomération et les communes du périurbain proche, par opposition aux systèmes de déplacement des habitants du centre de l'agglomération et des banlieues. Elles mettent en évidence la variété des stratégies de mobilité des ménages, mais aussi la diversité des territoires périurbains et de leurs ressources, en fonction de leur distance aux pôles d'emplois, de leur densité d'habitat et d'emploi, de leur maillage par des polarités secondaires dont les niveaux d'équipement diffèrent, et des profils sociodémographiques des populations résidentes.

6 On peut donc se demander si le modèle de territoires éclatés se vérifie toujours pour les ménages périurbains franciliens, en particulier ceux résidant dans l'ouest de la région, où le desserrement des résidences et des emplois a été plus précoce, et ce qui a changé dans leurs comportements de mobilité. Peut-on repérer une tendance au recentrage sur des pôles secondaires, de proximité? À travers ces analyses, on interroge la structuration des couronnes périurbaines à une échelle infra-métropolitaine, leur degré d'autonomie par rapport aux pôles urbains et la pertinence de la notion de bassin de vie pour décrire les systèmes de déplacements aux portes d'une très grande agglomération, particulièrement riche en équipements de niveau supérieur. 
Illustration 1 - L'ouest francilien : pôles urbains et espaces périurbains en 2010

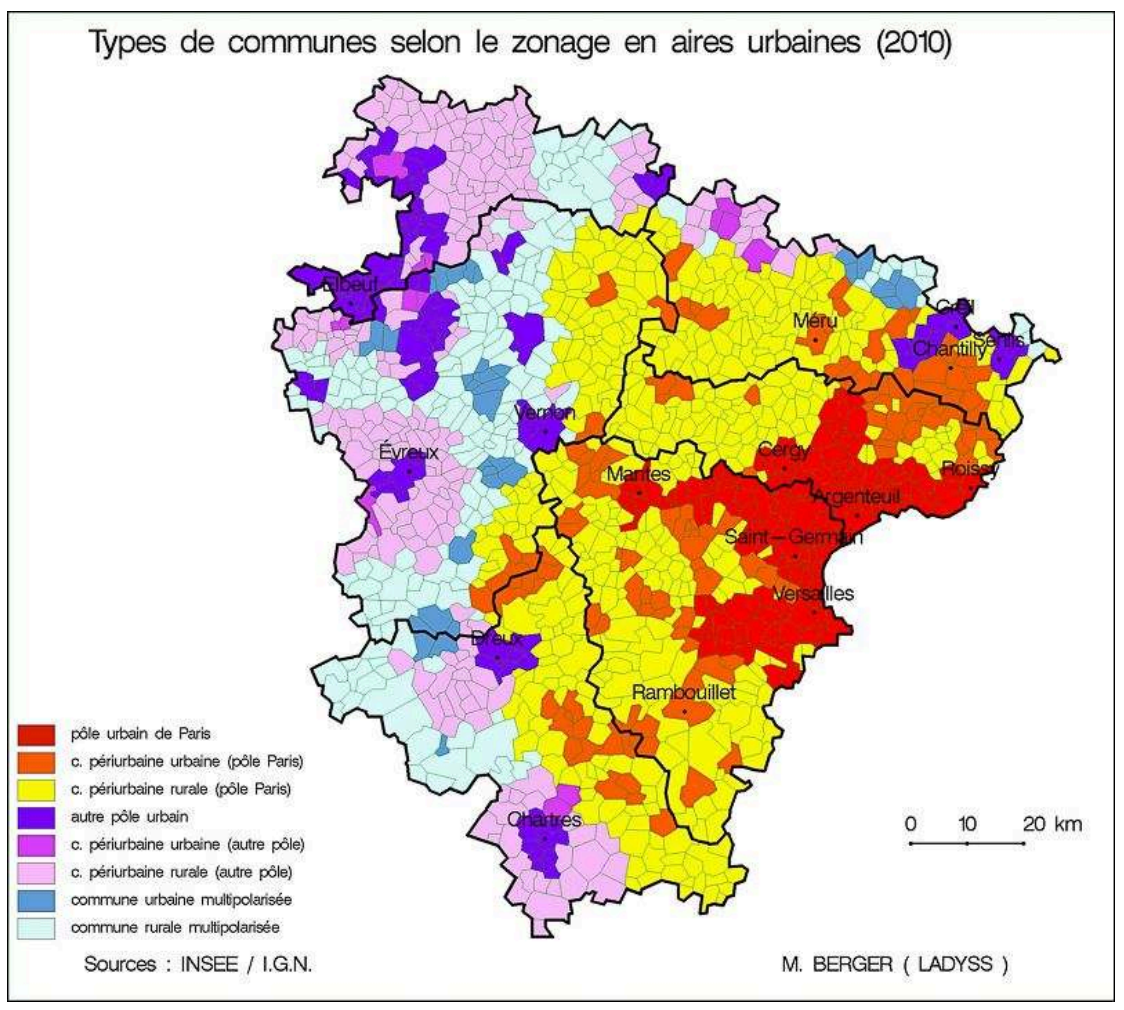

Source : INSEE, 2010 ; auteur : M. Berger.

7 Les couronnes périurbaines délimitées par l'INSEE incluent des villes petites et moyennes dans l'orbite de pôles d'emplois plus importants. Sur les 1299 communes comprises dans le périmètre d'étude, 1055 sont classées en 2010 comme périurbaines et regroupent plus d'1 million d'habitants. 177 d'entre elles sont urbaines au sens de l'Insee, et abritent un peu plus d'un demi-million d'habitants, soit une part équivalente à celle des communes périurbaines rurales, qui sont 5 fois plus nombreuses. La moitié des communes périurbaines de l'ouest francilien comptent moins de 500 habitants, et un tiers des périurbains de la zone vivent dans des communes de moins de 1000 habitants.

\section{Sources et méthodes}

\section{Sur les navettes domicile-lieu de travail}

Les distances des navettes domicile-travail ont été calculées à vol d'oiseau entre chefslieux de commune. On a affecté aux actifs travaillant dans leur commune de résidence une distance égale au rayon de cette commune (plafonné à $3 \mathrm{~km}$ ). Seuls les trajets inférieurs à $250 \mathrm{~km}$ à vol d'oiseau ont été retenus, les distances supérieures étant considérées comme relevant de pratiques de double résidence et non comme des déplacements quotidiens. Les calculs ont été effectués à partir du fichier-détail du recensement de 2006 (qui regroupe les collectes annuelles de 2004 à 2008) pour lequel nous avons pu croiser navettes domicile-travail et mobilités résidentielles (cf. infra), contrairement à d'autres sources plus récentes. 
9 Pour simuler les durées des navettes au recensement de 2006, on a utilisé les vitesses moyennes calculées à partir de l'Enquête Globale Transport (EGT) de 2010, en tenant compte des modes de transport utilisés. Il s'agit du mode de déplacement principal, à l'exclusion des rabattements, les durées incluant les attentes et les recherches de stationnements. L'EGT 2010 a porté sur un peu plus de 20000 personnes résidant en Îlede-France ayant effectué un déplacement domicile-travail le jour de l'enquête, dont environ 3000 périurbains. Les vitesses moyennes ont été calculées par mode et par zone, en utilisant le découpage établi par l'IAU-IdF qui divise l'île-de-France en 7 zones en fonction de la densité de la population et des emplois ( $\mathrm{cf}$ infra), soit 49 zones croisant origine et destination. Pour les actifs résidant et/ou travaillant hors île-de-France, on a retenu les vitesses moyennes des zones les plus externes.

10 L'objectif était de réaliser une comparaison entre actifs résidant dans les différentes zones, avec un échantillon plus large (plus de 500000 observations, représentant 1,6 million d'actifs au RP 2006) que celui de l'EGT, permettant une approche spatiale fine, au niveau communal. Les résultats obtenus dans cette simulation ne peuvent toutefois être comparés directement à ceux de l'EGT, en particulier parce que cette dernière, contrairement au recensement, ne prend en compte que les déplacements effectifs le jour de l'enquête, et exclut les actifs travaillant à domicile.

\section{Sur les autres motifs de déplacements}

11 L'étude des déplacements pour des motifs autre que le travail s'appuie sur les données des Enquêtes Globales Transport de 1976, 1983, 1991, 2001 (DRIEA) et 2010 (STIF, DRIEA). Pour l'année 2010, l'échantillonnage porte sur 18000 ménages franciliens, soit 43000 personnes (cf. La Mobilité en Ile-de-France STIF, OMNIL DRIEA, 2013). La région a pour cela été découpée en une centaine de secteurs. En grande couronne, ils regroupent généralement un pôle urbain, sa périphérie ainsi que les communes rurales qui l'entourent (cf. illustration 3). Ce zonage permet d'étudier les différences territoriales comme certains contrastes est-ouest. Ces secteurs ne suivent cependant pas les limites de l'agglomération: un même secteur peut ainsi contenir à la fois une partie périurbaine et une partie située en frange d'agglomération.

12 Afin de rendre compte de la structure du territoire et des formes urbaines, l'analyse s'est également appuyée sur le découpage morphologique réalisé à l'IAU-IdF (cf. Bertrand et Dugué, 2007, et illustration 2), fondé sur deux critères : d'une part, la proportion d'espaces urbanisés, et d'autre part, la densité humaine sur les espaces construits qui s'appuie sur la notion d'intensité des fonctions urbaines (résidentielles ou économiques). Ce découpage différencie sept zones franciliennes : quatre zones sont situées à l'intérieur de l'agglomération (le centre, les communes denses, les communes majoritairement urbanisées et les franges de l'agglomération). Trois autres zones sont situées en dehors de l'agglomération et permettent ainsi de distinguer trois morphologies différentes du périurbain : les communes principales des agglomérations périurbaines (comme Rambouillet, Houdan, Maule, Bonnières-sur-Seine,...), les communes secondaires (comme Ablis, Saint-Hilarion, La Queue-les-Yvelines, Bréval, Orgerus,...) et enfin les communes rurales. Les communes des agglomérations périurbaines sont classées comme principales si la somme population résidente + emplois est supérieure à 5 000, comme secondaires en deçà. 
Enfin, l'offre d'équipements a été prise en compte au travers d'une typologie en 6 classes synthétisant le niveau global d'équipement des communes franciliennes (cf. Mangeney, 2014 et illustration 3). Cette typologie reflète l'étendue de la gamme des équipements et services présents sur la commune dans différents domaines : champ de la petite enfance, de l'éducation et de la formation, champ sanitaire, des commerces, des équipements sportifs et des équipements culturels. Les communes de niveau 6 ont un éventail d'équipements suffisamment large pour couvrir toute la gamme des équipements et services de chacune de ces thématiques (par exemple, pour le domaine éducatif, celles qui disposent de services de garde de jeunes enfants, d'écoles, de collèges, de lycées mais également de sites d'enseignement supérieur). À l'inverse, les communes de niveau 1 sont assez mal équipées et leurs équipements ne couvrent que la partie la plus courante et la moins spécialisée de chaque thématique (pour reprendre l'exemple du domaine éducatif, il s'agit là des communes n'ayant aucune école ou n'ayant que des écoles maternelles ou élémentaires comme équipements d'enseignement).

\section{Illustration 2 - Découpage morphologique 2008}

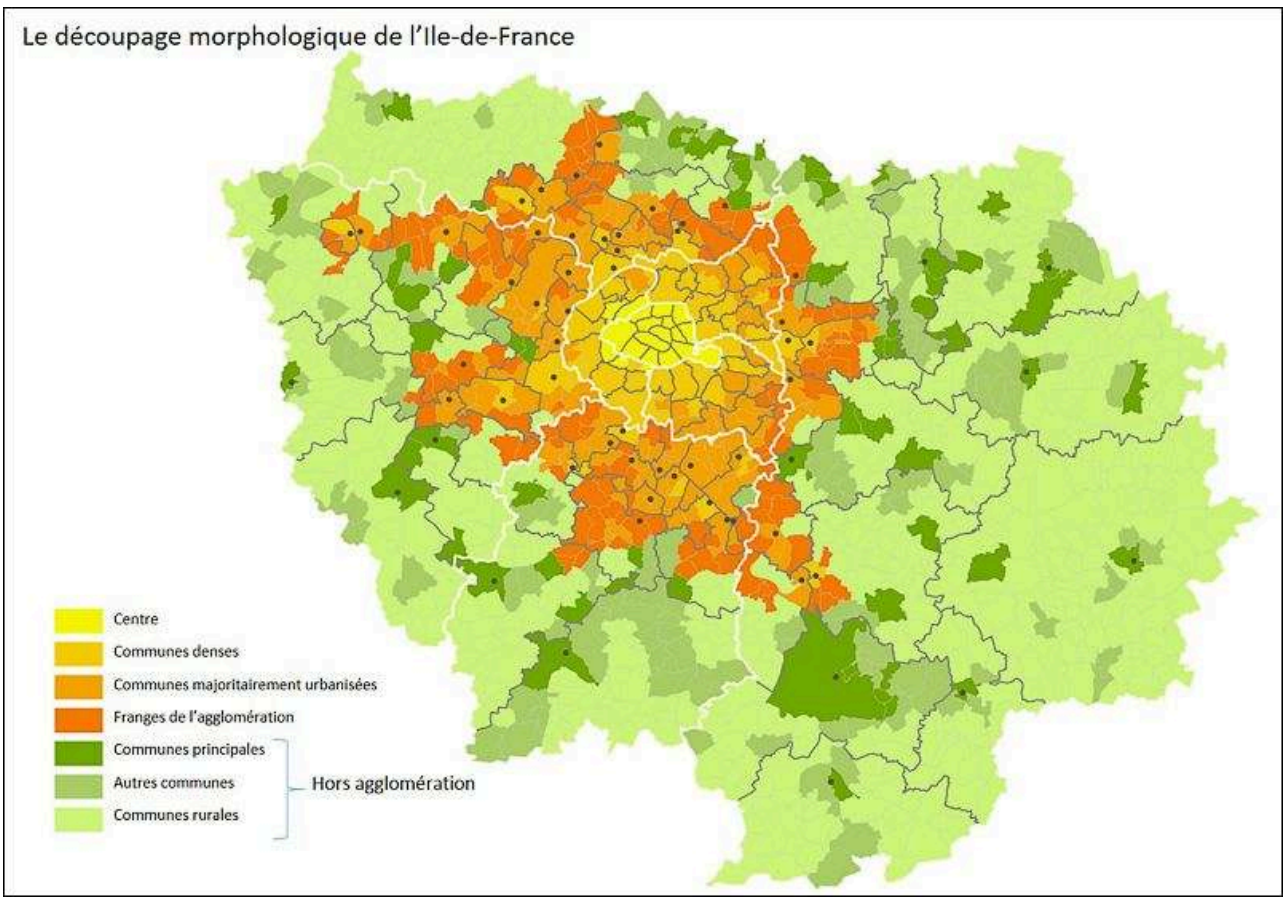

Auteur: Bertrand J., IAU -IdF. 
Illustration 3 - Typologie d'équipements des communes

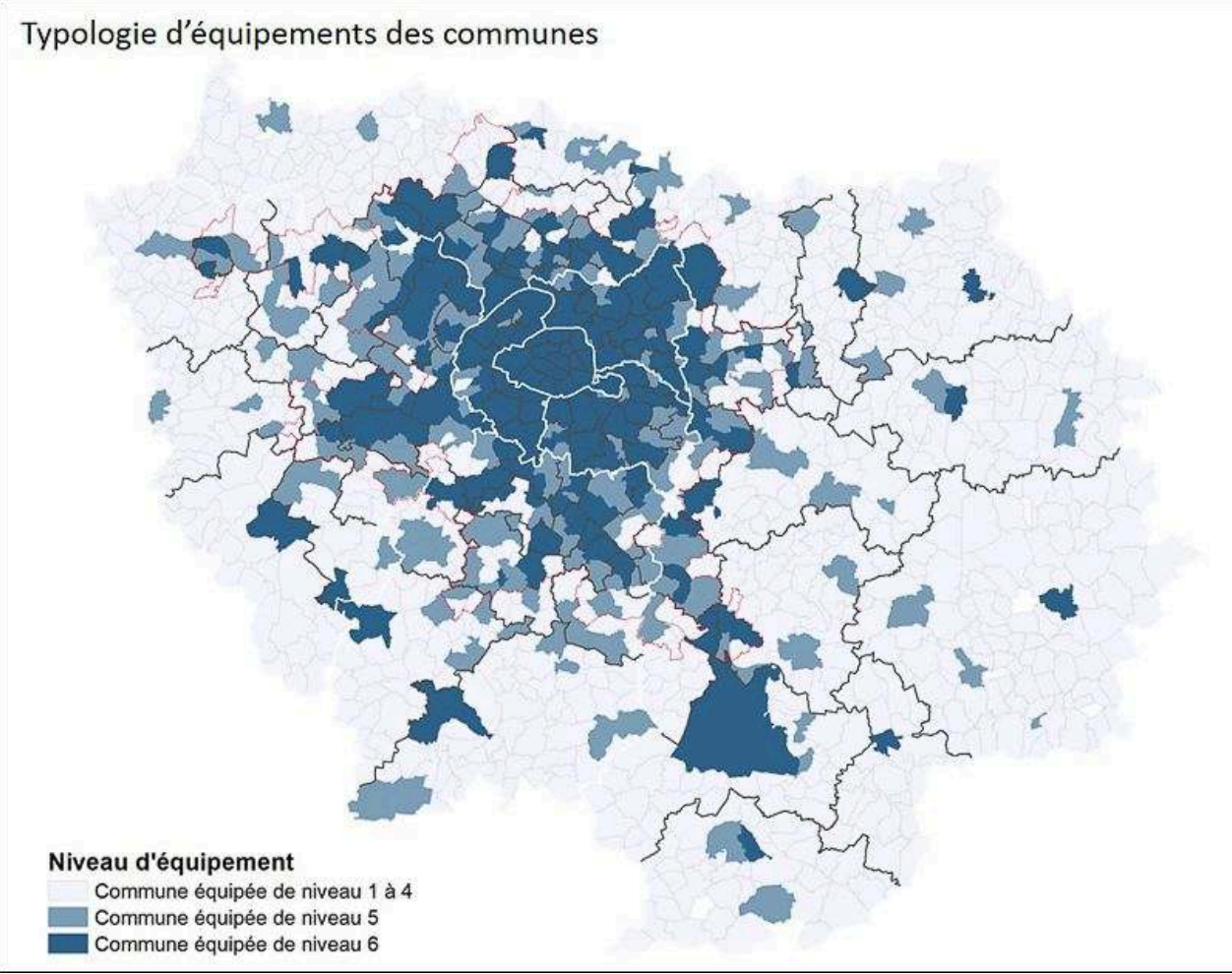

Auteur : Mangeney C. 2014, IAU-IdF

\section{Les navettes domicile-travail : principal facteur d'éclatement des espaces pratiqués?}

Si les périurbains effectuent en moyenne des navettes plus longues que les habitants de l'agglomération parisienne et ceux des autres pôles urbains, il faut nuancer ce constat à la fois au regard des distances parcourues et des budgets temps qui y sont consacrés.

\section{Des distances de navettes longues, mais qui ont cessé d'augmenter}

La longueur des déplacements entre domicile et lieu de travail et le faible recours aux transports collectifs constituent une des principales critiques faites à la forme périurbaine en termes de durabilité. Les périurbains de l'ouest francilien parcourent, pour se rendre à leur travail, des distances trois fois plus longues, en moyenne, que les habitants de Paris intra-muros (18,5 km en 2006 au lieu de 5,8 km), et 2,3 fois plus importantes que celles des actifs résidant en banlieue proche (les 3 départements de Petite couronne) ou 1,5 fois plus longues que celles des habitants de la grande banlieue (la partie du pôle urbain de Paris située en Grande couronne). Mais si on compare périurbains et habitants de l'agglomération parisienne résidant à une même distance de Paris, l'écart se réduit, le rapport n'est plus que de 1,1 à 1,2, alors même que l'offre d'emploi sur place est nettement plus favorable pour les actifs du pôle urbain.

$\mathrm{Au}$ sein des espaces périurbains, la différence est sensible entre communes rurales et communes urbaines : les distances moyennes parcourues par les ruraux pour se rendre 
à leur travail sont nettement supérieures à celles des urbains, qui effectuent plus souvent des navettes courtes (moins de $5 \mathrm{~km}$ ), au sein de la commune de résidence ou vers des communes voisines (cf. tableau 1). D'autre part, les périurbains de Paris ont des déplacements plus longs en moyenne que ceux des autres couronnes périurbaines (cf. illustration 4a), la moitié des actifs effectuant des trajets de plus de $15 \mathrm{~km}$ : les pôles urbains autres que l'agglomération parisienne ont un recrutement nettement plus local, polarisant fortement leur environnement. Ceci s'explique pour partie par des niveaux de qualification de la main d'œuvre différents, mais aussi par l'offre de transports collectifs en Île-de-France, qui facilite les navettes longues. Si l'offre locale d'emploi favorise les actifs des communes urbaines qui effectuent plus souvent des trajets courts, l'existence fréquente d'une desserte par les transports collectifs élargit pour eux l'horizon des possibles, d'où une part importante de navettes longues.

Tableau 1- Les distances domicile-travail des actifs de l'ouest francilien en 2006

\begin{tabular}{|c|c|c|c|c|c|c|}
\hline Type de & \multirow{3}{*}{$\begin{array}{l}\text { Distance moyenne } \\
\text { des navettes }(\mathrm{km})\end{array}$} & $\% \quad$ d'actifs & \multicolumn{4}{|c|}{$\%$ de navettes } \\
\hline \multirow[t]{2}{*}{ de résidence } & & la commune & \multirow{2}{*}{$\begin{array}{l}\text { moins de } \\
5 \mathrm{~km}^{*}\end{array}$} & \multirow{2}{*}{$\begin{array}{l}5- \\
14 \mathrm{~km}\end{array}$} & \multirow{2}{*}{$\begin{array}{l}15 \mathrm{~km} \\
\text { ou plus }\end{array}$} & \multirow{2}{*}{$\begin{array}{l}\text { dont } \\
50 \mathrm{~km} \text { ou } \\
\text { plus }\end{array}$} \\
\hline & & de résidence & & & & \\
\hline $\begin{array}{l}\text { pôle urbain de } \\
\text { Paris** }\end{array}$ & 11,4 & 21,6 & 36,5 & 34,9 & 28,7 & 0,7 \\
\hline $\begin{array}{l}\text { couronne } \\
\text { périurbaine de } \\
\text { Paris }\end{array}$ & & & & & & \\
\hline $\begin{array}{l}\text { communes } \\
\text { urbaines }\end{array}$ & 18,5 & 22,1 & 30,0 & 21,8 & 48,2 & 5,3 \\
\hline $\begin{array}{l}\text { communes } \\
\text { rurales }\end{array}$ & 21,1 & 15,6 & 22,9 & 25,7 & 51,4 & 8,9 \\
\hline $\begin{array}{l}\text { autres pôles } \\
\text { urbains }\end{array}$ & 13,5 & 34,9 & 55,8 & 20,1 & 24,0 & 6,7 \\
\hline $\begin{array}{l}\text { autres couronnes } \\
\text { périurbaines }\end{array}$ & & & & & & \\
\hline $\begin{array}{l}\text { communes } \\
\text { urbaines }\end{array}$ & 15,4 & 23,4 & 35,7 & 35,8 & 28,5 & 7,4 \\
\hline $\begin{array}{l}\text { communes } \\
\text { rurales }\end{array}$ & 16,7 & 15,9 & 23,9 & 41,2 & 33,7 & 7,4 \\
\hline
\end{tabular}

*Y compris les actifs travaillant dans leur commune de résidence.

**Partie de l'agglomération parisienne située dans les départements des Yvelines et du Val-d'Oise.

Source: RP 2006, exploitation complémentaire. Les calculs par types de communes ont été effectués dans la délimitation 2010 des unités urbaines et des aires urbaines. 
17 Si on observe l'évolution des navettes des actifs périurbains (cf. tableau 2), on constate qu'à une très forte croissance des distances parcourues dans les années 1980 - la distance moyenne passant de $11 \mathrm{~km}$ en 1975 à 16,7 km en 1990, succèdent, dans les communes périurbaines comme dans le pôle urbain parisien, une inflexion dans les années 1990, puis une stabilisation dans les années 2000, malgré la hausse des qualifications des actifs $^{5}$ (Berger, 2004 ; Berger et al., 2014).

Tableau 2 - L'évolution des distances domicile-travail dans l'ouest francilien (1975-2006)

\begin{tabular}{|c|c|c|c|c|c|c|c|c|c|c|}
\hline \multirow{2}{*}{$\begin{array}{l}\text { Type de } \\
\text { commune de } \\
\text { résidence* }\end{array}$} & \multicolumn{2}{|l|}{1975} & \multicolumn{2}{|l|}{1982} & \multicolumn{2}{|l|}{1990} & \multicolumn{2}{|l|}{1999} & \multicolumn{2}{|l|}{2006} \\
\hline & moyenne & médiane & moyenne & médiane & moyenne & médiane & moyenne & médiane & moyenne & médiane \\
\hline \multicolumn{11}{|l|}{$\begin{array}{l}\text { couronnes } \\
\text { périurbaines }\end{array}$} \\
\hline $\begin{array}{l}\text { communes } \\
\text { rurales }\end{array}$ & 10,7 & 4,2 & 14,4 & 7,1 & 17,3 & 9,4 & 18,9 & 11,7 & 19,1 & 12,4 \\
\hline $\begin{array}{l}\text { communes } \\
\text { urbaines }\end{array}$ & 10,8 & 2,7 & 13,3 & 5,9 & 16,2 & 9,7 & 17,7 & 12,3 & 17,9 & 13,0 \\
\hline \multicolumn{11}{|l|}{ pôles urbains } \\
\hline $\begin{array}{l}\text { agglomération } \\
\text { de Paris** }\end{array}$ & 9,5 & 6,9 & 10,2 & 7,7 & 11,2 & 8,6 & 11,5 & 8,9 & 11,4 & 8,7 \\
\hline $\begin{array}{l}\text { autres pôles } \\
\text { urbains }\end{array}$ & 7,0 & 1,7 & 8,2 & 2,1 & 11,0 & 3,0 & 12,2 & 3,0 & 13,5 & 3,6 \\
\hline
\end{tabular}

*Délimitation 2010 des unités urbaines et des aires urbaines.

**Partie de l'agglomération parisienne située dans les départements des Yvelines et du Val-d'Oise.

Sources : RP 1975 (1/5) ; RP 1982 et 1990 (1/4) ; RP 1999 (exhaustif) ; RP 2006, exploitation complémentaire.

18 Par rapport à des observations concernant l'ensemble de la France, qui mettent en évidence la poursuite de l'allongement des navettes à un rythme ralenti par rapport aux années 1980-1990, on constate donc une évolution plus précoce dans l'ouest francilien, comme dans l'ensemble de la région (Bouleau et Mettetal, 2014) où la densification tend désormais à l'emporter sur l'étalement (Baccaïni et Sémécurbe, 2009 ; Louchart, 2009). Ceci s'explique pour une part par l'ampleur du desserrement des emplois au sein du pôle urbain parisien et une évolution favorable du rapport entre le nombre d'actifs résidents et l'offre locale d'emplois dans l'ensemble constitué par les couronnes périurbaines et les franges de l'agglomération parisienne. Avec retard par rapport à celle de la population, on y observe une forte croissance des emplois relevant de l'économie présentielle, la densification de la population conduisant à la diversification de l'offre de commerces et de services.

19 On constate aussi que si les navettes des actifs résidant hors aire urbaine de Paris s'allongent toujours, les distances moyennes parcourues par ceux qui résident dans les cantons $\mathrm{du}$ Bassin parisien et travaillent en Île-de-France n'augmentent plus: 
l'augmentation de la portée des navettes ne s'explique donc qu'en partie par une intégration croissante dans les bassins d'emploi franciliens. La part des navettes à destination de Paris et de la Petite couronne diminue dans toutes les zones, témoignant d'une autonomisation croissante par rapport au centre du pôle parisien. L'extension des bassins d'emploi semble avoir atteint ses limites, l'aggravation et la généralisation de la congestion pesant sur les budgets-temps des navettes domicile-travail.

Illustration 4a- Les distances moyennes des navettes domicile-travail en 2006

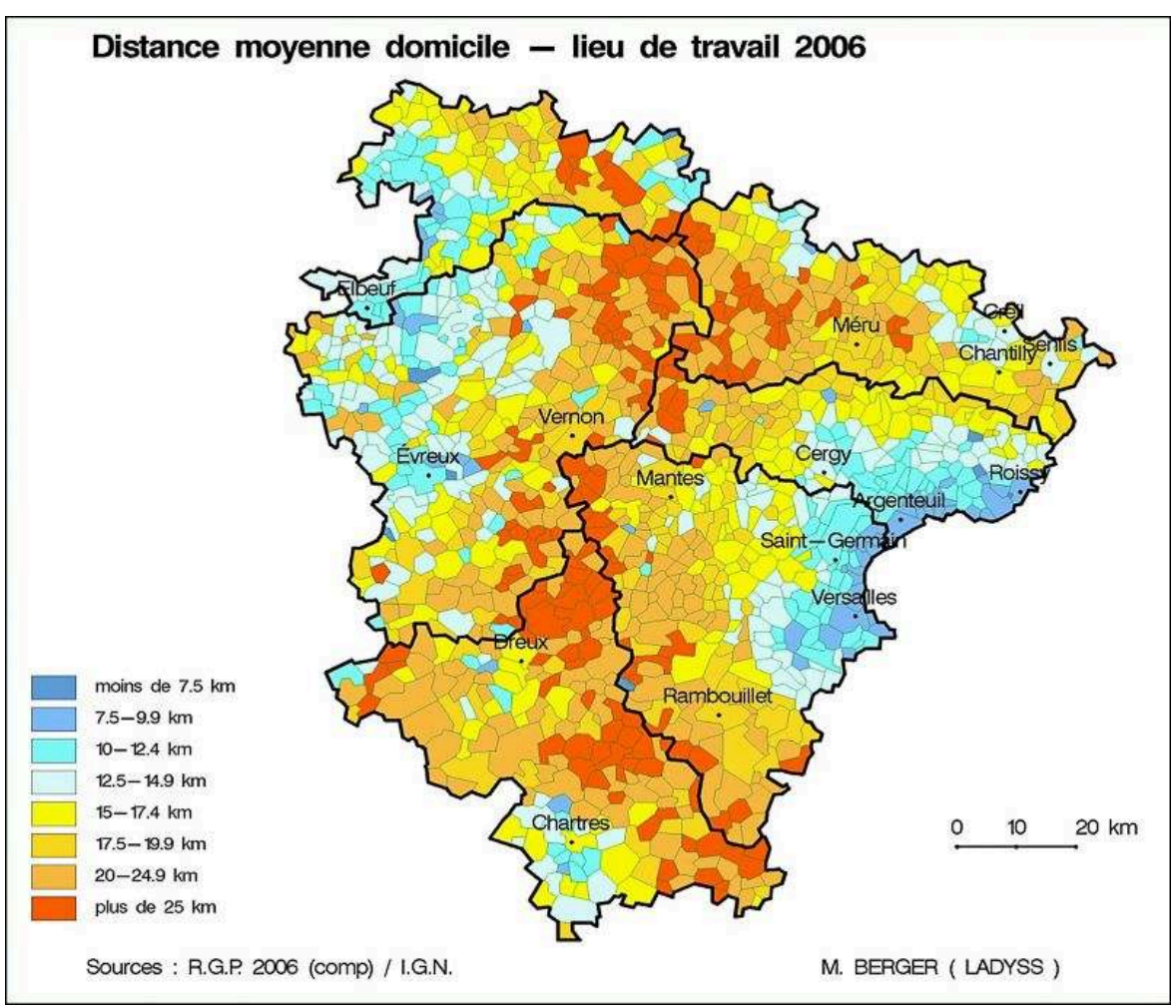

Source : INSEE, RP 2006 ; auteur : M. Berger

\section{Les budgets-temps des périurbains : proches de ceux des habitants des franges de l'agglomération}

Avec une durée moyenne des trajets domicile-travail de $42 \mathrm{mn}$, le budget-temps des périurbains de l'aire urbaine de Paris (cf. illustration $4 \mathrm{~b}$ ) est à peine supérieur à celui des habitants de la grande banlieue (39 $\mathrm{mn}$ pour ceux qui résident dans l'agglomération parisienne, dans les Yvelines et le Val-d'Oise), et il en est de même pour les périurbains plus lointains (33 $\mathrm{mn}$ ) par rapport aux habitants des autres pôles urbains $(32 \mathrm{mn})$. Se déplaçant dans des zones moins denses, plus fréquemment en voiture qu'en transports en commun, ils bénéficient de vitesses plus élevées, d'où des durées comparables à celles des urbains proches. Dans la couronne parisienne, la moitié des périurbains ont des navettes de moins d'une demi-heure, tout comme les habitants de l'agglomération, et 3 actifs périurbains sur 10 ont des trajets inférieurs à $15 \mathrm{mn}$, comme leurs voisins urbains. Les déplacements de plus de $60 \mathrm{mn}$ ne concernent qu'un quart des actifs périurbains franciliens, à peine plus que dans l'agglomération en Grande couronne, et sont nettement plus rares hors aire urbaine de Paris (un actif sur 7 ou 8), où il s'agit 
souvent de ménages récemment installés venus d'île-de-France, où ils ont conservé (temporairement ?) leur emploi. En effet, les périurbains ont tendance à changer de lieu de travail pour réduire leurs déplacements, à la fois parce qu'ils demeurent longtemps dans des logements dont ils sont propriétaires; parce que de nouvelles opportunités d'emploi s'ouvrent à proximité de leurs résidences, dans des communes qui se densifient et s'équipent ; et parce qu'avec le temps, ils apprennent à connaître les ressources de leur voisinage.

Illustration 4b - Les durées moyennes des navettes domicile travail en 2006

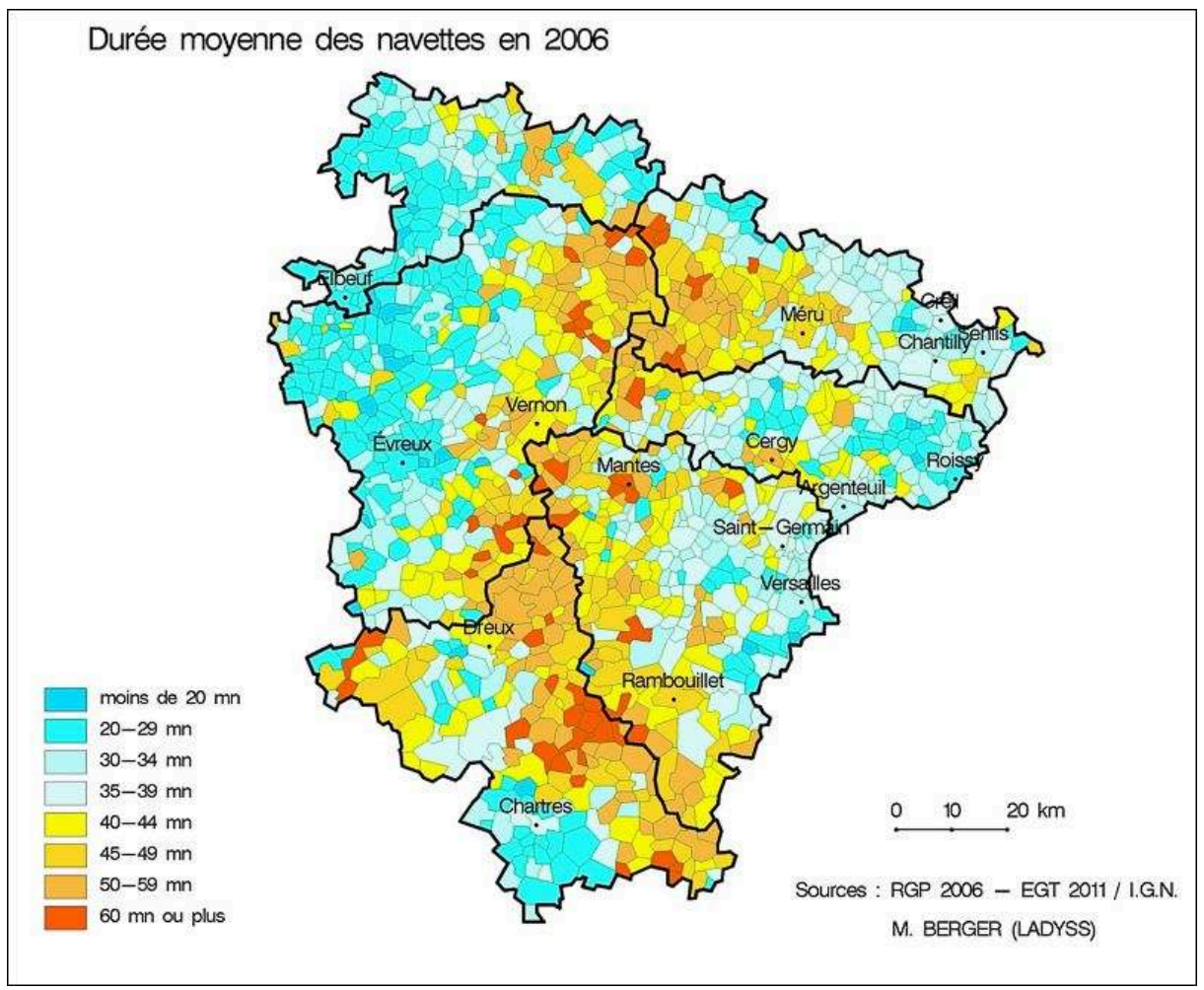

Source : INSEE, RP 2006/ EGT 2011 ; auteur : M. Berger.

\section{Les plus anciens installés: des navettes plus courtes}

21 Les distances domicile-travail des périurbains varient en fonction de la localisation de leur résidence, de leur catégorie socioprofessionnelle, et de leur sexe, mais aussi de leurs mobilités résidentielles. Comme l'ont montré des recherches précédentes portant sur l'ensemble de l'île-de-France (Berger, 2004), les périurbains de l'ouest francilien ayant effectué récemment une migration ont en moyenne des navettes plus longues $(21,3 \mathrm{~km})$ que ceux qui n'ont pas changé de logement $(17,4 \mathrm{~km})$, ou qui ont déménagé dans la même commune $(15 \mathrm{~km})$. Les distances entre domicile et lieu de travail mettent en évidence des arbitrages différents dans les stratégies résidentielles des ménages en fonction des budgets-temps qu'ils jugent acceptables. Ensuite, une partie d'entre eux change d'emploi: des ajustements des distances de navettes interviennent après l'installation dans une nouvelle commune, plus fréquemment pour les femmes que pour les hommes. 
22 Au sein des couples biactifs résidant dans le périurbain de l'ouest francilien, on s'est intéressé aux relations entre la distance des navettes et la durée d'installation du ménage dans le logement. Pour les conjointes, la part des navettes courtes (moins de $5 \mathrm{~km}$ ) augmente régulièrement, de $19 \%$ pour les actives installées depuis moins de 2 ans, à $37 \%$ pour celles qui occupent le logement depuis plus de 20 ans, alors que pour les hommes, l'écart est nettement plus restreint (de $19 \%$ à $30 \%$ ). À l'inverse, celle des navettes longues ( $15 \mathrm{~km}$ ou plus) passe de $50 \%$ pour les femmes installées depuis moins de deux ans à $28 \%$ pour celles qui habitent le logement depuis 20 ans ou plus (mais seulement de $56 \%$ à $41 \%$ pour les hommes). Si on mesure, toutes choses égales par ailleurs quant à l'âge, à la catégorie socioprofessionnelle, à la structure familiale (nombre d'enfants) et à la localisation résidentielle (distance à Paris et type de commune) l'ajustement des distances de navettes en fonction de l'ancienneté d'installation dans le logement, les méthodes de régression logistique confirment que pour les hommes des couples biactifs périurbains, la durée d'installation joue très peu sur la probabilité d'effectuer des navettes longues (plus de $15 \mathrm{~km}$ ), alors que pour leurs conjointes, elle diminue régulièrement quand l'ancienneté dans le logement augmente. Comparé aux actives qui ont emménagé depuis moins de 2 ans, le rapport de chances (odd's ratio) est de 0,66 pour celles qui sont installées depuis plus de 20 ans (mais de 0,92 pour les hommes). La réduction des trajets domicile-travail apparaît 2 à 5 ans après le déménagement et est particulièrement nette pour les employées, qui profitent du développement de l'offre locale d'emplois présentiels, moins ample dans des catégories plus qualifiées. Ceci confirme les observations faites dans la Megalopolis nordaméricaine (Hanson et Pratt, 1991) sur le rôle du bouche à oreille et des relations sociales locales dans la recherche d'emploi par les actives les moins diplômées. Mais la proximité domicile-travail n'est pas pour autant synonyme de pratiques de déplacement plus vertueuses: dans l'espace périurbain, 2 navettes de moins de $5 \mathrm{~km}$ sur 3 sont effectuées en voiture.

23 L'évolution des distances des navettes témoigne donc de changements de comportements, souvent liés à un ancrage résidentiel de plus en plus long et facilités par le desserrement des emplois vers les marges de l'agglomération; mais aussi parce que le système de mobilités dans la métropole parisienne a atteint ses limites en termes de portée, de budgets-temps et d'efficacité des déplacements et tend à évoluer vers une fragmentation et une polarisation autour de noyaux secondaires, comme le confirme l'analyse des autres types de déplacements.

\section{Les autres motifs de déplacements}

Même si la part des retraités augmente rapidement dans les couronnes périurbaines ${ }^{6}$, le déplacement vers le lieu de travail reste le déplacement le plus structurant des mobilités des ménages périurbains, qui construisent leur vie quotidienne autour de leurs déplacements "obligés». Les trajets professionnels, auxquels s'ajoutent les déplacements domicile-études des enfants, structurent leur territoire de vie. Le déplacement domicile-travail est généralement le déplacement le plus long en distance, le plus fréquent, et de ce fait le plus contraint. Les autres motifs de déplacements, de loisirs, d'achats, d'accompagnements, pour santé ou démarches administratives s'ajustent ensuite, en fonction de l'offre, c'est-à-dire des équipements disponibles dans les polarités avoisinantes mais également des stratégies individuelles. L'analyse des 
pratiques de mobilité et de leurs évolutions au regard de la morphologie urbaine met en évidence la diversité des modes d'habiter dans le périurbain, et la similitude avec les usages des habitants des franges de l'agglomération ou de zones plus denses, ainsi que les spécificités des mobilités des ménages périurbains résidant dans l'ouest francilien par rapport au reste de la région.

\section{Des territoires de vie étendus en distance mais pas nécessairement en temps}

Malgré une saturation croissante des réseaux routiers dans de nombreux secteurs du périurbain, l'usage massif de l'automobile ${ }^{7}$ permet encore de parcourir des distances importantes en des temps beaucoup plus courts que dans l'agglomération. Cette fluidité dessine des proximités assez étendues lorsqu'on les compare à celles de l'agglomération, alors que les durées de déplacement sont comparables. Cependant, pour la première fois depuis les années 1970, la vitesse moyenne d'un déplacement dans le périurbain est en baisse (Bouleau et Mettetal, 2014). Cette évolution est le résultat de plusieurs phénomènes qui se cumulent: la congestion de plus en plus courante dans de nombreux secteurs $d u$ périurbain, la règlementation $d u$ stationnement en ville (le temps de recherche d'un stationnement est inclus dans la durée du déplacement), le plus grand respect des limitations de vitesse, ainsi que l'aménagement de nombreuses voiries limitant la vitesse des véhicules particuliers. Cette baisse s'accompagne pour la première fois d'une diminution de la part modale de la voiture pour les déplacements domicile-travail : elle passe de $76 \%$ à $73 \%$ entre 2001 et 2010 alors qu'elle n'avait fait que croître depuis les années 70 .

Ainsi, alors que les portées des déplacements peuvent être trois fois plus longues dans les communes rurales que dans les communes denses, les durées moyennes ne varient quasiment pas, quelle que soit la densité du tissu urbain (cf. illustration 5). L'analyse des distances et durées au travers du découpage morphologique révèle par ailleurs l'écart entre les communes principales du périurbain et les communes rurales, où les distances sont de 2 à 3 fois plus longues. À l'image de sa morphologie, le périurbain francilien est un territoire aux pratiques extrêmement variables : dans ses communes principales, les distances sont très proches de celles pratiquées à l'intérieur de l'agglomération, tous motifs de déplacements confondus y compris les trajets domiciletravail, alors que dans les communes rurales, les pratiques sont beaucoup plus distendues. 
Illustration 5 - Portées (distances à vol d'oiseau) des déplacements en km en moyenne et médiane et durée moyenne en minutes selon le découpage morphologique de la commune de résidence de l'individu

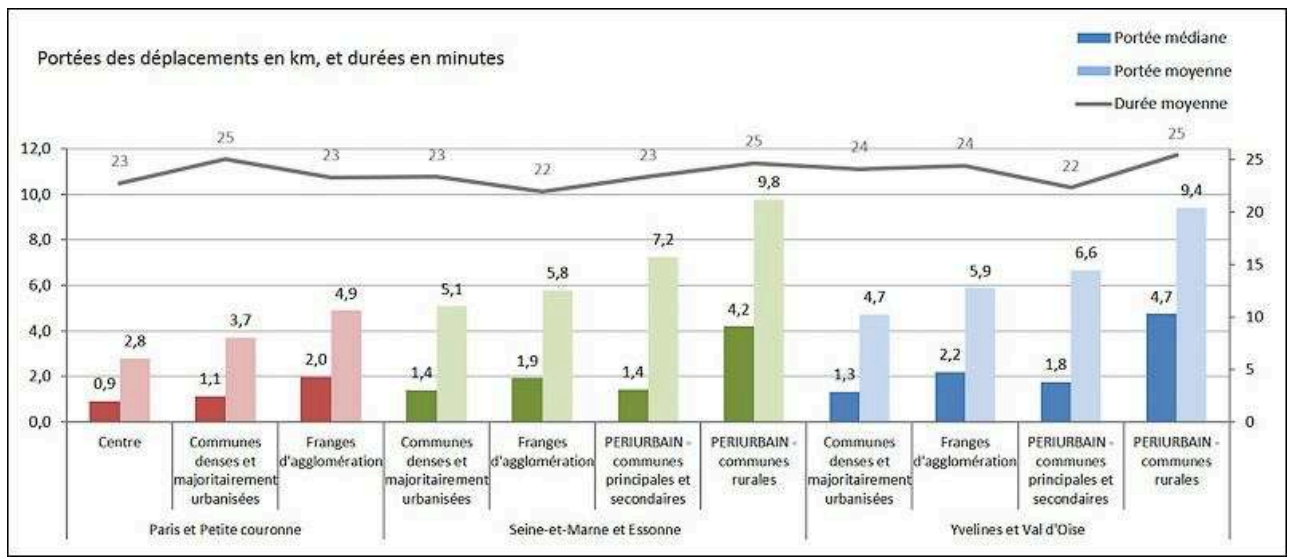

Source : EGT 2010, STIF DRIEA OMNIL ; auteur : M. Bouleau, IAU-IdF.

\section{Des pratiques très différentes selon les zones et selon les individus}

L'éloignement entre les activités réalisées et le domicile, en moyenne (illustration 6) et en médiane (Illustration 7) permet d'évaluer la diversité des pratiques particulièrement importante dans les espaces périurbains (Cailly ET Dodier, 2007). Quel que soit le motif du déplacement, l'éloignement moyen augmente progressivement au fur et à mesure que l'on s'éloigne du centre de l'agglomération. Certaines disparités territoriales apparaissent, en particulier concernant les loisirs ou les affaires personnelles (santé et démarches administratives). Dans de nombreux secteurs du périurbain, $50 \%$ des déplacements sont réalisés à moins de $2 \mathrm{~km}$ du domicile. Cet écart entre moyenne et médiane montre la variabilité des comportements. La moyenne, tirée par des valeurs fortes mais rares, tend à masquer le comportement le plus fréquent.

Il apparait ainsi, pour chaque domaine d'activité, 3 types de territoires :

- Les territoires fortement ancrés dans la proximité ont à la fois un éloignement moyen faible et un éloignement médian faible. Il s'agit de secteurs où la proximité est l'usage le plus fréquent et où les comportements atypiques sont peu nombreux. À côté du centre de l'agglomération, quelques secteurs périurbains (Torcy, Les Ulis ou Fontainebleau) ont des pratiques très locales en ce qui concerne les achats.

- Les territoires hétérogènes ont un éloignement médian faible mais une moyenne élevée. Si le comportement le plus fréquent reste l'usage de la proximité et des ressources locales, d'autres modes de vie, plus distants, cohabitent. Le Vexin, les secteurs de Plaisir, Provins, Melun ou Meaux sont dans cette situation en ce qui concerne les achats. Alors qu'un déplacement pour achat sur deux y est réalisé à moins de $2 \mathrm{~km}$, l'éloignement moyen varie de 5 à $10 \mathrm{~km}$. Ces écarts peuvent concerner des ménages différents : les personnes âgées par exemple utilisent souvent plus l'offre locale (Desjardins et Mettetal, 2012). Mais il peut également s'agir de différents types d'achats d'un même ménage: les achats quotidiens pouvant se contenter d'une offre locale et les achats plus exceptionnels recherchant une offre plus lointaine. Enfin, cette dispersion peut être géographique pour des secteurs regroupant un pôle urbain dense et des tissus ruraux très peu équipés. 
- Les territoires des grandes distances ont des éloignements médians et moyens élevés. Les secteurs de L'Isle-Adam, de Limay en périphérie de Mantes, d'Étampes et de Nemours sont dans ce cas pour les achats, les secteurs de Coulommiers ou de l'Isle-Adam pour les loisirs.

Illustration 6 - Éloignement moyen entre la destination du déplacement et le lieu de résidence selon le motif du déplacement

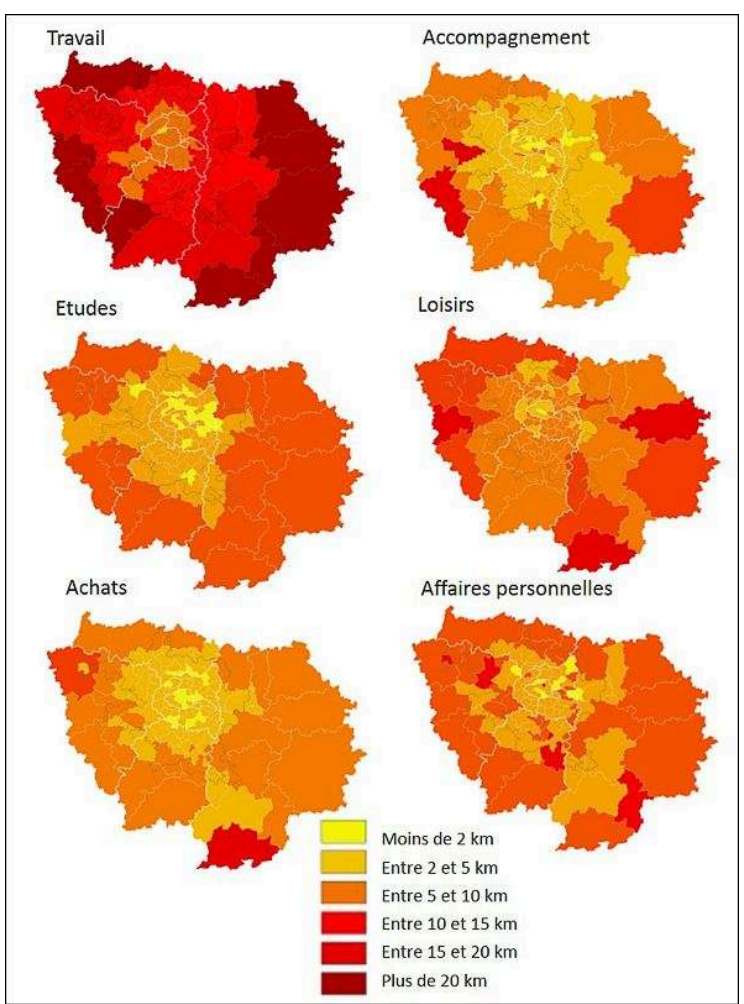

Source : EGT 2010, STIF DRIEA OMNIL ; auteur : M. Bouleau, IAU-IdF. 
Illustration 7 - Éloignement médian entre la destination du déplacement et le lieu de résidence selon le motif du déplacement

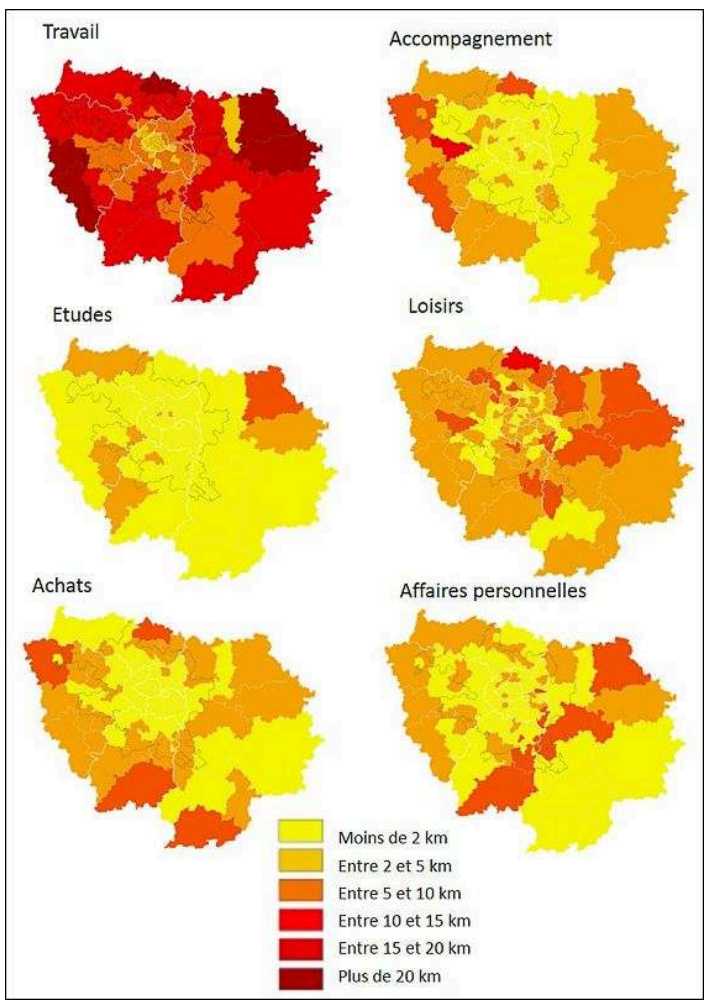

Source : EGT 2010, STIF DRIEA OMNIL ; auteur : M. Bouleau, IAU-IdF.

\section{Une proximité systématiquement investie}

Quel que soit le territoire vécu, même dans les «territoires des grandes distances », la proximité reste la première destination des déplacements autres que le travail. Afin de rendre compte des arbitrages des individus, les destinations des déplacements non contraints (réalisés pour d'autres motifs que travail et études) ont été distinguées en 6 catégories exclusives :

- La proximité : regroupe les déplacements dont la destination se situe dans la commune de résidence de l'individu ou dans un rayon de $500 \mathrm{~m}$ autour de son domicile.

- Le lieu de travail: concerne les déplacements dont la destination est située dans la commune du lieu de travail ou dans un rayon de $5 \mathrm{~km}$ autour de celui-ci. Lorsque le lieu de travail est situé à proximité du domicile, un déplacement vers le lieu de travail est considéré comme étant un déplacement de proximité.

- Paris : lorsque la destination du déplacement est Paris, hors proximité et lieu de travail. Les déplacements à Paris des personnes travaillant à Paris sont considérés comme des déplacements sur le lieu de travail.

- Commune de niveau 6 : regroupe les déplacements dont la destination est située dans une commune très bien équipée de niveau 6 (cf. supra), hors proximité, lieu de travail ou Paris.

- Commune de niveau 5 : concerne les activités réalisées dans une commune assez bien équipée de niveau 5 (cf. supra), hors proximité, lieu de travail ou Paris.

- Autres communes : les activités réalisées dans des communes moins bien équipées, hors proximité, lieu de travail ou Paris. 
30 sont réalisés à proximité (cf. illustration 8 ). Dans les communes principales du périurbain, la proximité concentre près de $50 \%$ des déplacements. Ce poids est plus fort en Seine-et-Marne et dans l'Essonne où il atteint $55 \%$, dépassant même les communes denses ou majoritairement urbanisées de ces départements. Dans le périurbain des Yvelines et du Val-d'Oise, le poids de la proximité est moins important dans les communes principales mais il est en revanche supérieur dans les communes secondaires. Ces différences montrent une hiérarchie un peu différente des pôles périurbains à l'est et à l'ouest. L'échelonnement semble plus structuré à l'Ouest. Les communes «secondaires» du périurbain des Yvelines et du Val-d'Oise restent polarisantes. L'usage de la proximité y est intermédiaire : plus important que dans les communes rurales, tout en n'atteignant pas le niveau des communes principales. En Seine-et-Marne et dans l'Essonne, ce niveau intermédiaire est moins présent. Les pôles principaux sont très structurants et peu concurrents entre eux en raison de leur éloignement. Leurs habitants utilisent donc très majoritairement les ressources locales, ils se rendent assez peu dans d'autres communes, même bien équipées. Les habitants des pôles secondaires en revanche n'utilisent pas plus leur proximité que ceux des communes rurales.

Enfin, il est intéressant de noter les rôles marginaux du lieu de travail et de Paris pour les activités non professionnelles. Ce sont en effet les communes bien équipées de niveau 6 , situées en dehors de la proximité du lieu de résidence et du lieu de travail, qui prennent, le cas échéant, le relais de la proximité, attirant entre 20 et $30 \%$ des déplacements.

Illustration 8- Répartition des déplacements autres que travail ou études (accompagnement, achats, loisirs ou autres motifs personnels) selon la typologie du lieu de destination et le niveau d'équipement des communes

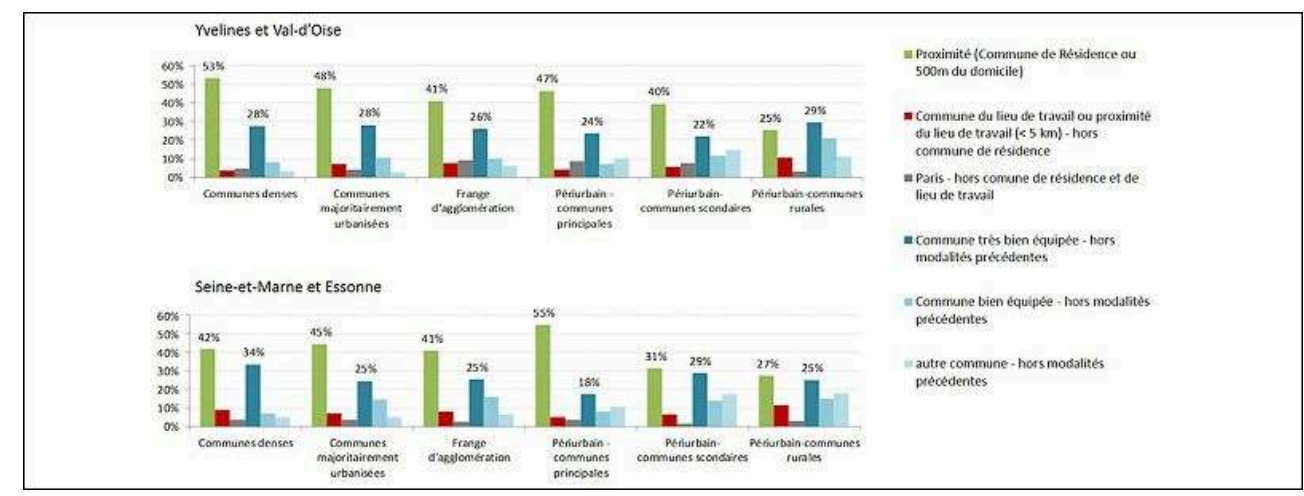

Sources : EGT 2010, STIF DRIEA OMNIL et Mangeney C. 2010 IAU-IdF ; auteur : M. Bouleau, IAU-IdF. 
Illustration 9 - Part des déplacements autres que travail ou études (accompagnement, achats, loisirs ou autres motifs personnels) réalisés dans la proximité du domicile (commune de résidence ou dans un rayon de $500 \mathrm{~m}$ à vol d'oiseau du domicile)

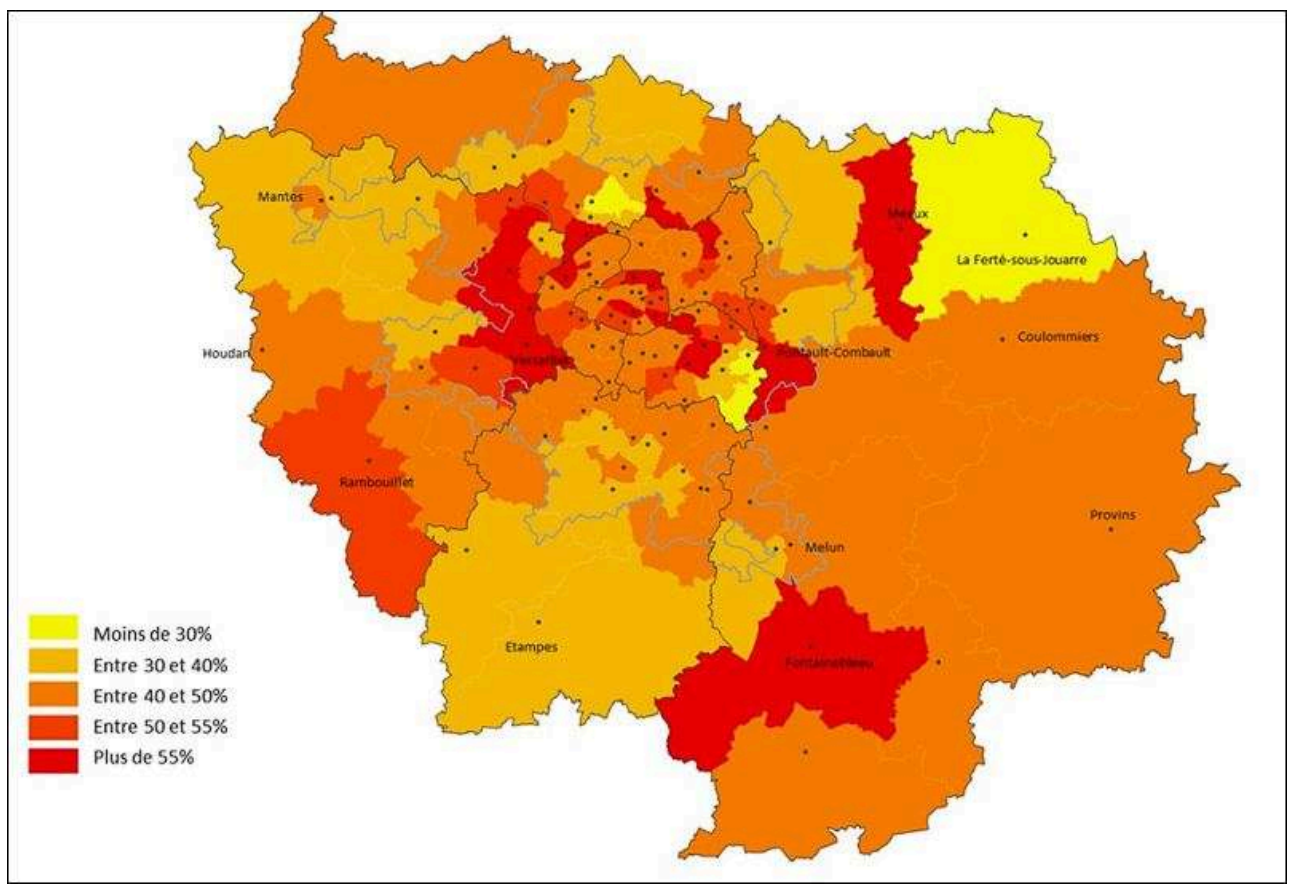

Source : EGT 2010, STIF DRIEA OMNIL ; auteur : M. Bouleau, IAU-IdF.

\section{Un renforcement de l'ancrage}

Depuis les années 1990, la maitrise de l'étalement urbain permet une évolution progressive des pratiques des habitants du périurbain, et fait apparaître un rapport plus «ancré » au territoire, qui s'illustre notamment par un recentrage des activités autour du lieu de résidence (Bonnin-Oliveira et al., 2012). Rompant avec le modèle centre-périphérie, les espaces périurbains atteignent une forme de maturité (Bouleau et Mettetal 2014; Berger, Aragau, Rougé, 2014). Le périurbain était, en effet, jusque dans les années 2000, la seule zone où les distances continuaient à croître fortement (alors qu'elles étaient stabilisées dans toute l'agglomération depuis les années 1970) entrainant un espace de vie de plus en plus étendu quel que soit le motif du déplacement, des activités les plus courantes aux plus exceptionnelles. En 2010, les distances se stabilisent enfin. La longueur moyenne d'un déplacement est même en légère baisse (cf. illustration 10). Pour la première fois, les périmètres vécus se reconcentrent, pour tous les motifs autres que le travail, faisant écho au désir d'ancrage exprimé par les habitants (cf. Bouleau et Mettetal, 2013).

Dans les Yvelines et le Val-d'Oise, l'éloignement entre les lieux d'activités et le domicile semble dorénavant stabilisé dans les polarités principales du périurbain (cf. illustration 11). Dans le périurbain seine-et-marnais, un recentrage est assez net après une période d'étalement plus marquée que dans le périurbain ouest. En 2010, dans l'ouest francilien, les habitants des communes rurales et ceux des polarités principales et secondaires du périurbain pratiquent des territoires de taille très similaire à ceux des franges d'agglomération. 
Illustration 10 - Évolution des portées moyennes des déplacements depuis 1976, selon la morphologie du lieu de résidence

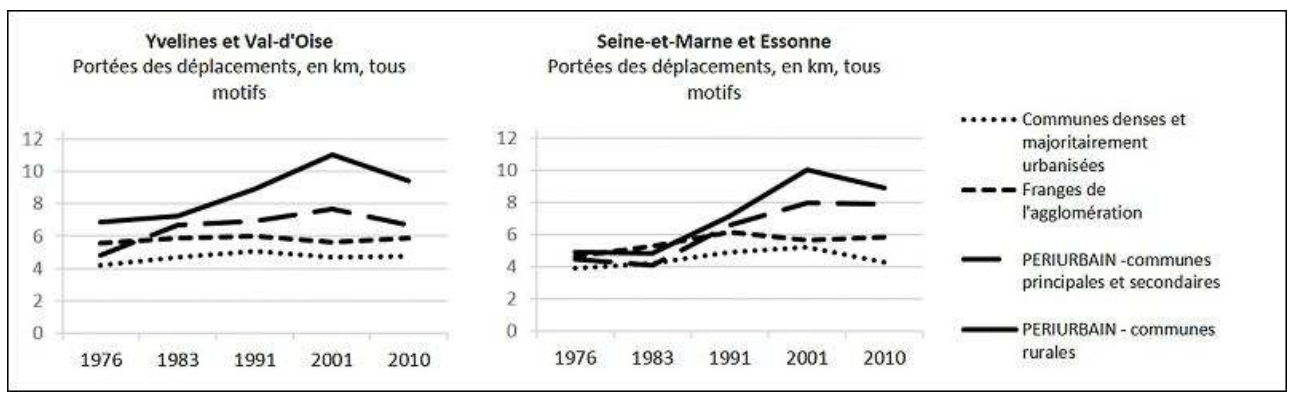

Sources : EGT 1976, 1983, 1991, 2001 DRIEA et EGT 2010, STIF DRIEA OMNIL ; auteur : M. Bouleau, IAU-IdF.

Illustration 11 - Évolution de l'éloignement moyen en km entre le lieu de destination des déplacements et le lieu de résidence selon le motif du déplacement, dans le périurbain francilien depuis 1976

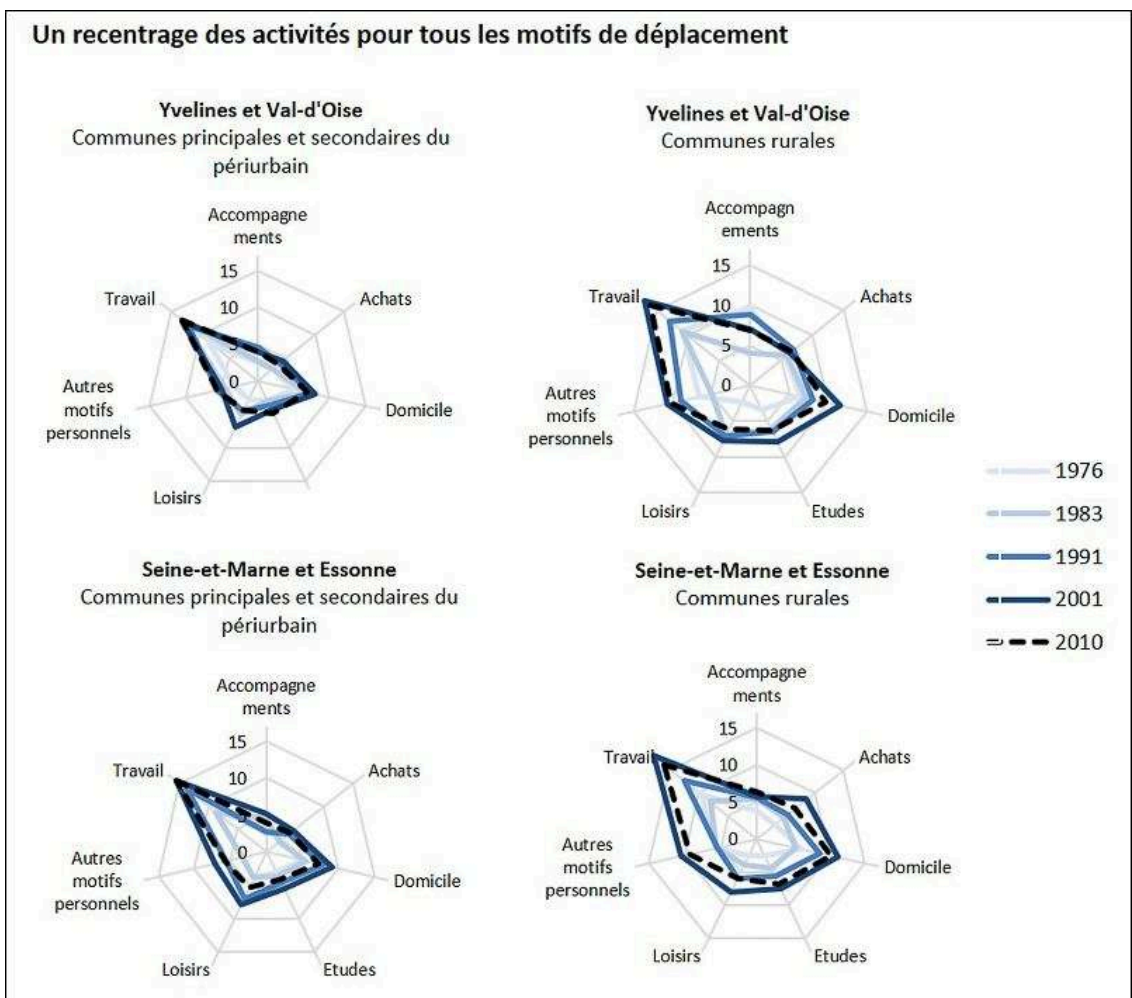

Sources : EGT 1976, 1983, 1991, 2001 DRIEA et EGT 2010, STIF DRIEA OMNIL ; auteur : M. Bouleau, IAU-IdF.

La diversité des pratiques dans le périurbain et la structuration différenciée des déplacements selon la morphologie de la commune de résidence et les aménités qu'elle offre s'observent-elles également au niveau micro-local? Des enquêtes auprès des ménages ${ }^{8}$ montrent un ancrage local relativement fort, et une utilisation diversifiée des différentes polarités du voisinage, en fonction des motifs, des envies, des dessertes, des mobilités. Elles confirment que la présence d'une centralité constituée ou la densité de l'offre locale en équipements et services ont une incidence sur la dispersion des déplacements mais aussi sur le niveau d'ancrage local, le lien que les habitants ont avec Paris, ou avec toute autre centralité voisine. C'est principalement pour des raisons 
médicales, de conduite aux aéroports, ou de loisirs et visites à des parents ou amis que se font les déplacements les plus longs, et les trajets en direction du centre de l'agglomération. Si la délimitation de "bassins de vie » regroupant l'ensemble des pratiques habitantes semble ici délicate, il apparait en revanche pertinent de lire le territoire d'après sa structuration en "pôles » (d'équipements, d'emploi...) emboîtés, selon différentes échelles de rayonnement, faisant écho à la lecture et à l'usage que les Franciliens ont eux-mêmes de leurs espaces de vie.

\section{Conclusion}

L'analyse des déplacements réalisés pour des motifs autres que le travail confirme les évolutions observées pour les navettes: une tendance qui semble maintenant bien dessinée à un recentrage sur des territoires de proximité, une utilisation accrue de ressources locales qui se diversifient et s'étoffent, de la part d'une population dont l'ancrage local se renforce. Ces ressources se distribuent dans l'espace périurbain et les franges de l'agglomération dans un système de «lieux " constitués chacun d'une ou plusieurs communes qui polarisent les déplacements. Ces polarités se hiérarchisent en fonction de leurs proximités, mais aussi des motifs de déplacements : certaines sont «multifonctionnelles » et drainent les déplacements quels que soient les motifs, alors que d'autres semblent plus spécialisées dans une fonction. Au sein des pôles principaux, les habitants, tout périurbains qu'ils soient, ont des habitudes de déplacements très ancrées dans la proximité, exploitant au maximum les ressources locales et parcourant des distances souvent plus courtes que ceux des franges de l'agglomération. Peut-on pour autant parler de véritables bassins de vie, de territoires effectivement partagés par tous les habitants d'un même ensemble de communes? Les périurbains utilisent l'ensemble des choix possibles en matière de commerces et de services, ils fréquentent tous les types de communes, mais pas les mêmes communes. Si la proximité est investie par tous, à moyenne échelle, les flux sont très éclatés car les territoires périurbains sont maillés par un réseau de polarités concurrentes, d'où un éclatement des pratiques spatiales, avec une fréquentation régulière des bords de ville, un rapport distancié et utilitaire à la grande ville.

Par ailleurs, les espaces périurbains apparaissent différenciés, notamment en fonction de la profondeur historique de leur développement : dans le périurbain yvelinois et vald'oisien, des polarités secondaires se sont progressivement constituées, faisant apparaitre des comportements intermédiaires entre les habitants des pôles principaux et ceux des communes rurales. Dans le périurbain plus récent de l'est francilien, le système est plus simple sans véritablement d'échelon intermédiaire entre des pôles principaux très polarisants et des communes rurales. Qu'il s'agisse des trajets domiciletravail ou des autres déplacements, les comportements des périurbains sont donc bien loin de répondre à un modèle unique. Au-delà des choix individuels, ils renvoient à la diversité croissante des espaces périurbains et de leurs ressources, en fonction de leur distance aux pôles d'emplois, de leur densité d'habitat et d'emploi, de leur maillage par des polarités secondaires dont les niveaux d'équipement diffèrent, ainsi que des profils sociodémographiques des populations résidentes. Ils témoignent des interrelations étroites entre formes d'implantation du peuplement et systèmes de mobilités quotidiennes. 


\section{BIBLIOGRAPHIE}

Aragau C., 2013. Le bassin de vie, un territoire porteur de ruralité aux marges de l'île-de-France. Norois, $n^{\circ} 229$, p. 7-20.

Armoogum J., Hubert J.-P., Roux S., Le Jeannic T., 2010. Plus de voyages, plus de kilomètres quotidiens : une tendance à l'homogénéisation des comportements de mobilité des Français, sauf entre ville et campagne. In La mobilité des Français, Panorama issu de l'enquête nationale transports et déplacements 2008, La Revue du CGDD, p. 5-23.

Baccaïni B., Sémécurbe F., Thomas G., 2007. Les déplacements domicile-travail amplifiés par la périurbanisation. Insee Première, $\mathrm{n}^{\circ} 1129,4 \mathrm{p}$.

Baccaïni B., Sémécurbe F., 2009. La croissance périurbaine depuis 45 ans : Extension et densification. Insee Première, $\mathrm{n}^{\circ}$ 1240, 4 p.

Berger M., 2004. Les périurbains de Paris. De la ville dense à la métropole éclatée. Paris, CNRS Éditions, $317 \mathrm{p}$.

Berger M., Aragau C., Rougé L., 2014. Vers une maturité des territoires périurbains ? EchoGéo [En ligne], 27 | 2014, mis en ligne le 02 avril 2014. URL : http://echogeo.revues.org/13683 ; DOI : $10.4000 /$ echogeo.13683

Bertrand J., Dugué R., 2007. Le nouveau découpage morphologique. Note aux utilisateurs. Paris, IAUIdF.

Bonnin-Oliveira S., Berger M., Jaillet M-C. (dir.), 2012. Les pôles secondaires dans la réorganisation des mobilités : maturité et durabilité des espaces périurbains? Rapport de recherche, PUCA, 210 p.

Bouleau M., Mettetal L., 2013. La mobilité dans le périurbain francilien, PODAM77. IAU-IdF, rapport de recherche, PREDIT (GO3), $110 \mathrm{p}$.

Bouleau M., Mettetal L., 2014. La mobilité dans le périurbain : désir d'ancrage et nouvelles proximités. Note Rapide $n^{\circ} 646$, IAU-IdF.

Bouleau M., Mangeney C., 2015, Déplacements et usages de la métropole, Coupes et découpes territoriales - Quelles réalité du Bassin de vie ? Les Cahiers, nº 172, IAU-IdF, p. 81-86.

Cailly L., Dodier R., 2007. La diversité des modes d'habiter des espaces périurbains dans les villes intermédiaires : différenciations sociales, démographiques et de genre. Norois, nº 205, p. 67-80.

Cavard J.-C., 1989. Par les villes et les villages. Données sociales Île-de-France, Insee, p. 49-53.

Cordobes S., Lajarge, R., Vanier M., 2010. Vers des périurbains assumés. Quelques pistes stratégiques pour de nouvelles régulations de la question périurbaine. Territoires $2040, \mathrm{n}^{\circ} 2$, Documentation Française, p. 21-32.

Desjardins X., Mettetal L., 2012. L'habiter périurbain face à l'enjeu énergétique. Flux, nº 89-90, p. 46-57.

Desplanques G., 1990. Les villes les plus fréquentées de la grande Couronne. L'T̂le-de-France en mouvement. Reclus Mode d'emploi, $\mathrm{n}^{\circ}$ 16, p. 337-343.

Gallez C., Orfeuil J.-P., 1998. Dis-moi où tu habites, je te dirai comment tu te déplaces. In Pumain D. et Mattéi M.-F. (dir.), Données Urbaines 2, Paris, Anthropos, p. 157-164. 
Grimal R., 2012. Des mobilités plus homogènes ou plus diversifiées ? Économie et Statistique, $\mathrm{n}^{\circ}$ 457-458, p. 13-34.

Hubert J.-P., Delisle F., 2010. L'allongement des déplacements quotidiens contribue à l'émergence d'espaces urbains multipolaires, tandis que la mobilité baisse au centre des grandes agglomérations. In La mobilité des Français. Panorama issu de l'enquête nationale transports et déplacements 2008, La Revue du CGDD, p. 49-64.

Louchart P., 2009. Île-de-France 2006 : 870000 Franciliens de plus qu'en 1990. Note rapide $n^{\circ} 466$, Iau-IdF, 6 p.

Mangeney C., 2014. Les polarités d'équipements et services en île-de-France. Rapport d'étude IAU IdF, $100 \mathrm{p}$.

Motte-Baumvol B., 2008. La dépendance automobile pour l'accès des ménages aux services : Le cas de la grande couronne francilienne. Revue d'Économie Régionale et Urbaine, n 5, p. 897-919.

Nicolas J.-P., Verry R., Longuar Z., 2012. Évolutions récentes des émissions de $\mathrm{CO}_{2}$ liées à la mobilité des Français : analyser les dynamiques à l'œuvre grâce aux enquêtes nationales Transports de 1994 à 2008. Économie et Statistique, n 457-458, p. 161-183.

Poulot M., Aragau C., Berger M., Rougé L., Mettetal L., 2014. Les territoires périurbains : de l'hybridation à l'intensité. Rapport final PUCA, LAVUE-Mosaïques, PRODIG, ESO, IAU-Idf, 320 p. En ligne : halshs-01145733, version 1.

Vanier M., 2011. La périurbanisation comme projet. Métropolitiques, 23 février 2011. http:// www.metropolitiques.eu/La-periurbanisation-comme-projet.html

\section{NOTES}

1. Ces travaux ont été menés dans le cadre de deux recherches réalisées pour le PUCA (BonninOliveira et al., 2012 ; Poulot et al., 2014).

2. Ces travaux ont été réalisés dans le cadre d'une recherche coordonnée par l'IAU-IdF portant sur les bassins de vie (M. Bouleau, C. Mangeney, 2015) s'inscrivant dans le cadre des débats parlementaires autour de la loi Notre.

3. Le volume annuel moyen de construction des maisons individuelles est passé de 15000 entre 1975 et 1990 à 7800 entre 1990 et 2003.

4. En 2006, la durée moyenne d'installation dans le logement était de 15 ans pour l'ensemble des ménages périurbains. Près de $3 / 4$ (73\%) d'entre eux résidaient déjà dans la même commune 5 ans auparavant. La moitié occupe le même logement depuis au moins 10 ans, un sur 4 depuis 23 ans. La distance moyenne des déménagements est passée de 25,7 km en 1990 à 22,6 km en 2006, avec une augmentation sensible de la part des mobilités dans un rayon de 5 à $14 \mathrm{~km}$.

5. Les cadres représentent en $200617 \%$ des actifs périurbains (au lieu de $10 \%$ en 1982) et leurs navettes sont 1,5 fois plus longues que celles des ouvriers.

6. La part des personnes de référence retraitées et inactives de plus de 60 ans est passée de $23 \%$ en 1982 à $29 \%$ en 2006.

7. En $201085 \%$ des déplacements en modes mécanisés et $63 \%$ de l'ensemble des déplacements sont réalisés en voiture dans le périurbain francilien. Sur la base des déplacements autres que domicile-travail, la voiture représente $61 \%$ des déplacements tous modes confondus.

8. 250 entretiens semi-directifs ont été réalisés auprès de ménages résidant dans des bourgs, des petites villes ou des communes rurales, principalement dans 4 secteurs: autour de Maule et Guerville (Yvelines), dans le Vexin (Val-d'Oise), dans la Communauté de Communes du Pays Houdanais (Yvelines et Eure-et-Loir), ainsi que dans les cantons de Pacy et Saint-André (Eure). 


\section{RÉSUMÉS}

Les périurbains sont souvent considérés comme uniformément contraints à des mobilités de longue distance. L'analyse des évolutions récentes des mobilités des périurbains de l'ouest francilien (à partir des recensements de population et des Enquêtes Globales Transport) met en évidence une stabilisation des distances moyennes et des budgets-temps des navettes domiciletravail, une plus grande recherche de proximité dans les comportements d'achat quotidiens, une utilisation plus fréquente et plus différenciée des ressources locales, ainsi que la diversité des territoires périurbains et de leurs ressources. Elle souligne également les continuités entre les franges de l'agglomération et les communes du périurbain proche, par opposition aux systèmes de déplacement des habitants du centre de l'agglomération.

Residents of periurban areas are often considered to be forced to travel long distances. The observation of recent trends in mobility in the outer suburbs and periurban fringe of Paris (based on censuses and transport surveys) highlights a stabilization of average distances and travel-time budgets of commuting trips. A growing tendency towards shorter shopping trips, greater diversity of these areas and their resources, and a clear relationship between local supply in terms of amenities and services, and household mobility strategies are revealed. Observations highlight the diversity of these periurban areas - particularly those located in the west of the Paris region - and also display a continuity in mobility patterns between the fringes and the periurban suburbs which are clearly distinguished from the urban core.

\section{INDEX}

Keywords : periurban fringe, commuter, daily mobility, travel-time budget, level of amenities and services, île-de-France

Mots-clés : espace périurbain, mobilité quotidienne, navette domicile-travail, budget-temps, niveau d'équipement, île-de-France

\section{AUTEURS}

\section{MARTINE BERGER}

Martine Berger, martine.berger@univ-paris1.fr, est professeur émérite à l'Université Paris 1 et membre de l'UMR Prodig. Elle a publié récemment :

- Berger M., Aragau C., Rougé L., 2014. Vers une maturité des territoires périurbains? Développement des mobilités de proximité et renforcement de l'ancrage dans l'ouest francilien, EchoGéo [En ligne], 27/2014, mis en ligne le 2 avril 2014. http://echogeo.revues.org/13683 - Aragau C., Berger M., Rougé L., 2013. Les mobilités des périurbains de l'ouest francilien : vers une quête de proximité ?, Géo-Regards, 6, 53-67.

- Berger M., 2013. Entre mobilités et ancrages : faire territoire dans le périurbain, Métropolitiques, 11 janvier 2013. http://www.metropolitiques.eu/Entre-mobilites-et-ancrages-faire.html.

\section{MIREILLE BOULEAU}

Mireille Bouleau, Mireille.Bouleau@iau-idf.fr, économètre, est chargée d'études à l'IAU îdf. Elle a publié récemment :

- Bouleau M., Mettetal L., 2013. La mobilité dans le périurbain francilien, PODAM77. IAU-IdF, rapport 
de recherche, PREDIT (GO3), $110 \mathrm{p}$.

- Bouleau M., Mettetal L., 2014. La mobilité dans le périurbain : désir d'ancrage et nouvelles proximités. Note Rapide $n^{\circ} 646$, IAU-IdF.

- Bouleau M., Beaufils S., 2014. La mobilité dans le Vexin français. Diagnostic de la demande de déplacements, IAU-IdF, $57 \mathrm{p}$.

\section{CATHERINE MANGENEY}

Catherine Mangeney, Catherine.Mangeney@iau-idf.fr démographe-urbaniste, est chargée d'études à l'IAU-IdF. Elle a publié récemment :

- Bouleau M. et Mangeney C. (sous la coord.de), 2015. Coupes et découpes territoriales, quelle réalité du bassin de vie ?, Les Cahiers de l'IAU-IdF, n 172, $176 \mathrm{p}$.

- Mangeney C., 2014. Les polarités d'équipements et services en Île-de-France. Rapport d'étude IAU-IdF, $100 \mathrm{p}$.

- Albe-Tersiguel S., Mangeney C. (sous la coord. de), 2011. Équipements et services, la métropole au quotidien, Les Cahiers de l'IAU-IdF, $\mathrm{n}^{\circ} 157,88 \mathrm{p}$. 